\title{
X-Ray Powder Diffraction Patterns for Certain $\beta$-Lactam, Tetracycline and Macrolide Antibiotic Drugs
}

\author{
S. Thangadurai, ${ }^{* 1}{ }^{\dagger}$ J. T. Abraham, ${ }^{* 2}$ A. K. Srivastava, ${ }^{2}$ M. Nataraja Moorthy, $* 3$ \\ S. K. SHUKLA, ${ }^{* 2}$ and Y. ANJANEYULU $* 4$ \\ *1 Department of Geology and Mining, Guindy, Chennai-32, India \\ *2 Central Forensic Science Laboratory, Directorate of Forensic Sciences, Hyderabad-13, India \\ *3 Universiti Sains Malaysia, 16150 Kubang Kerian, Kelantan, Malaysia \\ *4 Institute of Science and Technology, JNTU, Kukadpally, Hyderabad-72, India
}

\begin{abstract}
$\mathrm{X}$-ray powder diffraction (XRD) data for eight $\beta$-lactam viz., ampicillin sodium, ampicillin trihydrate, penicillin $\mathrm{G}$ procaine, benzathine penicillin, benzyl penicillin sodium, cefalexin, cefotaxime sodium and ceftriaxone sodium; three tetracyclines viz., doxycycline hydrochloride, oxytetracycline dihydrate and tetracycline hydrochloride; and two macrolide viz., azithromycin and erythromycin estolate antibiotic drugs were obtained using a powder diffractometer. The drugs were scanned from Bragg angles $(2 \theta)$ of $10^{\circ}$ to $70^{\circ}$. The obtained data were tabulated in terms of the lattice spacing $(\AA)$ and relative line intensities $\left(I / I_{\mathrm{I}}\right)$. This new information may be useful for identifying these drugs from confiscated materials, which has been frequently encountered in forensic laboratories.
\end{abstract}

(Received December 23, 2004; Accepted April 18, 2005)

\section{Introduction}

$\beta$-Lactam antibiotic drugs, i.e., penicillin and cephalosporins, are related chemically by the presence of a four-membered ring containing a nitrogen atom adjacent to a carbonyl group (a $\beta$ lactam ring). Antibacterial activity and stability (penicillin $v s$. cephalosporins) is defined by the stereospecificity and geometry of the fused side ring. Side chains and stereochemistry play an important role in determining their antibacterial activity. The basic structure of the penicillin nucleus includes a $\beta$-lactam ring fused through nitrogen and adjacent tetrahedral carbon to a second heterocycle, which in natural penicillin is a fivemembered thiazolidine ring (Fig. 1).

The nucleus common to all penicillins is the fused thiazolidine-amino- $\beta$-lactam, which consists of L-cysteine and D-valine. The free carboxyl group of the thiazolidine is essential for therapeutic effectiveness. In 1940 several researchers embarked on a scientific endeavor to identify and isolate this class of compounds, and as the result of this important pioneering work the penicillins were instrumental during World War II for treating battle casualities.

The cephalosporins are the second family of $\beta$-lactam antibiotics, discovered after the penicillins, and there are numerous parallelisms between the two families. All of the cephalosporins are semi-synthetic derivatives of the natural cephalosporin $\mathrm{C}$ antibiotic. Cephalosporins are $\beta$-lactam antibiotics with the same fundamental structural requirements as penicillin (Fig. 1). The heterocyclic ring fused to $\beta$-lactam ring is six-membered dihydrothiazine in cephalosporins. The fused rings in $\beta$-lactams are not coplanar, but folded along the $\mathrm{C}-\mathrm{N}$ bond common to both rings, less markedly in cephalosporins

† To whom correspondence should be addressed.

E-mail: kalankathanga@hotmail.com/gmail.com than in penicillins.

There are strong historical, biological and chemical similarities between the cephalosporins and the penicillins. The common feature is the $\beta$-lactam ring, which is hydrolyzed by $\beta$ lactamase. These similarities are key to developing analytical methods for this class of drugs.

The tetracyclines (TCs) comprise a family of both bio-

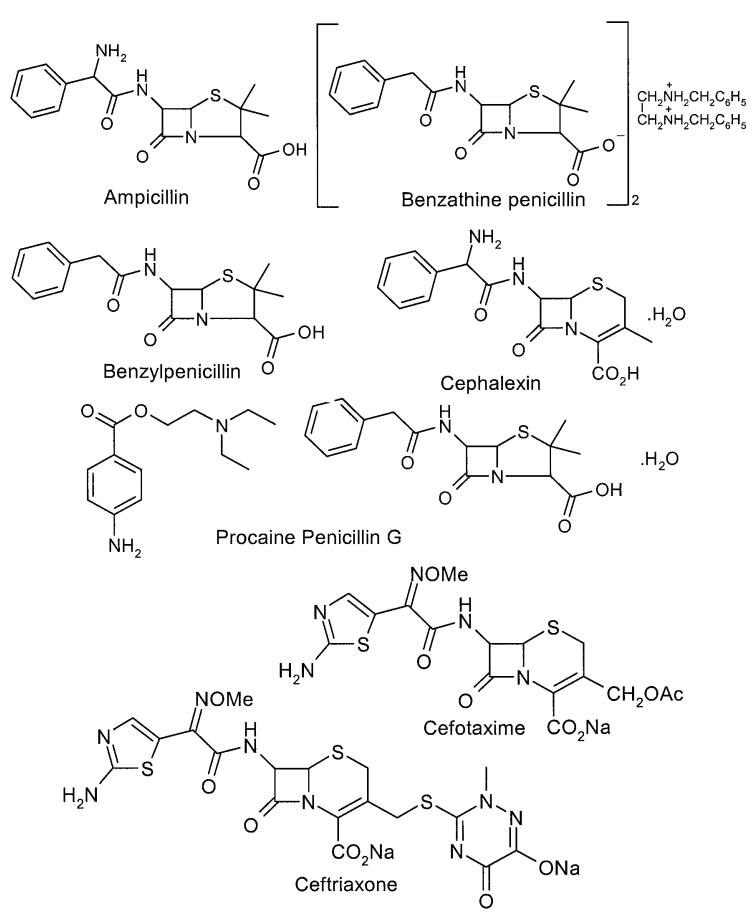

Fig. 1 Molecular structure of $\beta$-lactam antibiotics. 


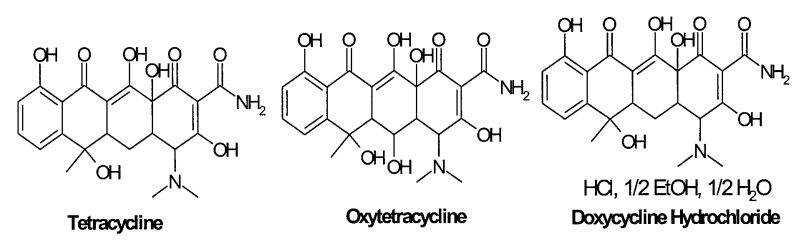

Fig. 2 Molecular structure of tetracycline antibiotics.

synthetic and semi-synthetic derivatives, which are characterized by an unusual antimicrobial background. However, these compounds are not active against the common cold or fungi. The first TC (7-chlortetracycline) was discovered in 1948, and it was the second compound to be developed after chloramphenicol as a broad-based antibiotic.

Chemically, TCs contain an octahydronaphthacene ring skeleton, consisting of four fused rings (Fig. 2). All of the TCs have a common skeleton, but differ in their substituents, which has a significant influence on their individual solubilities. The TCs are amphoteric and can be manufactured as both acidic and basic salts. ${ }^{1}$

The macrolide antibiotics most commonly used in agriculture are tylosin and erythromycin. Macrolide antibiotics are used in veterinary medicine to treat respiratory diseases, or as feed additives to promote growth. These antibiotics are lipophilic molecules with a central lactone ring bearing 12 to 16 atoms, to which several amino or neutral sugars are bound. ${ }^{2}$

Although erythromycin (EM) was widely used until the 1980 s, it is unstable to acid, and its pharmacokinetics after oral administration is highly variable. Recently developed semisynthetic derivatives of EM, such as azithromycin (AZM), clarithromycin (CAM) and roxithromycin (RXM), are stable to acid and more extensively distributed to tissues. The generalized structure is a highly substituted monocyclic lactone (aglycone) to which is attached one or more saccharide units amino or deoxy sugar, glycosidically linked to hydroxyl groups on either the aglycone or another saccharide. They may be divided into 12-, 14- and 16-membered aglycone ring macrolides (Fig. 3).

The development of new compounds with the potential for drug abuse necessitates a continuous accumulation of analytical data in the forensic laboratory. Also, the identification of excipients and adulterants in drug samples provides a database that can be used for intelligence purposes. A correlation of cases can provide investigative leads as well as being supporting evidence in conspiracy cases. The purpose of the present paper is to present X-ray powder diffraction data, which are not available in the literature. Included in the paper are data on compounds where more complete and accurate tabulations than those already published have been obtained.

The XRD powder method has proven to be very effective and specific in the identification of crystalline substances. For example, different hydrates of the same compound, as well as polymorphic forms, have completely different X-ray diffraction patterns. Compounds in mixtures can be identified without separation. $^{3}$ This method is a powerful technique for the identification of crystalline solid phases. ${ }^{4}$ Every crystalline solid phase has a unique XRD pattern, which can form the basis for its identification. In a powder mixture, each crystalline phase produces its pattern independently of the other constituents in the mixture. ${ }^{5}$ The technique is unique, since it combines absolute specificity with a high degree of accuracy. ${ }^{6}$ Despite these attributes, the method finds very limited application for the evaluation of drug product quality.

There have been a number of applications of XRD analysis to

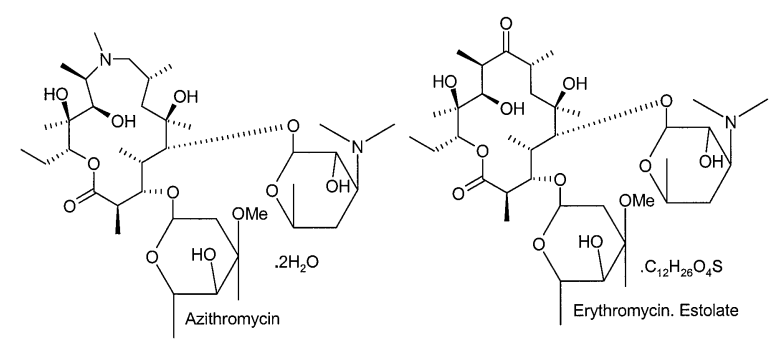

Fig. 3 Molecular structure of macrolide antibiotics.

organic materials. Among the many compounds that have been characterized by powder XRD methods are solid aromatic hydrocarbons. ${ }^{7,8}$ Recently, X-ray powder diffraction has also been found useful in elucidating the structures and in the identification of natural products, ${ }^{9}$ analgesics, ${ }^{10,11}$ amines ${ }^{12,13}$ and antibiotics. ${ }^{14-16}$ Also, it has become one of the most powerful tools in forensic analysis. ${ }^{17,18}$

As is true with all analytical procedures, XRD is most powerful when used in conjunction with other techniques, such as emission spectroscopy, X-ray fluorescence and chemical analysis. An important advantage of X-ray diffraction over these and other techniques is that the obtained results give information about the materials as they occur in the sample, rather than only about the individual elements or ions present. It is often the only satisfactory method for distinguishing among polymorphs, or detecting a compound in the presence of others containing the same elements.

The powder XRD technique was used here to characterize eight $\beta$-lactam antibiotic drugs, viz., ampicillin sodium, ampicillin trihydrate, penicillin $G$ procaine, benzathine penicillin, benzyl penicillin sodium, cefalexin, cefotaxime sodium and ceftriaxone sodium; three tetracyclines viz., doxycycline hydrochloride, oxytetracycline dihydrate and tetracycline hydrochloride; and two macrolide viz., azithromycin and erythromycin estolate antibiotics in terms of interplanar $d$ spacings and line intensities relative to the intensity of the strongest line.

The powder XRD method has not been suggested in USP, ${ }^{19}$ $\mathrm{BP}^{20}$ and $\mathrm{IP}^{21}$ for identifying these antibiotic drugs, but infrared (IR) absorption, thin-layer chromatography (TLC), highperformance liquid chromatography (HPLC) and polarographic methods are suggested. A review of the literature reveals that no considerable attention has been paid to powder XRD in identification and determination of these antibiotics. The highlight of this work was to provide X-ray powder diffraction data for the above mentioned antibiotic drugs.

\section{Experimental}

\section{Samples}

Pure samples of ampicillin sodium $\left(\mathrm{C}_{16} \mathrm{H}_{18} \mathrm{~N}_{3} \mathrm{NaO}_{4} \mathrm{~S}\right)$, ampicillin trihydrate $\left(\mathrm{C}_{16} \mathrm{H}_{19} \mathrm{~N}_{3} \mathrm{O}_{4} \mathrm{~S} \cdot 3 \mathrm{H}_{2} \mathrm{O}\right)$, penicillin $\mathrm{G}$ procaine $\left(\mathrm{C}_{16} \mathrm{H}_{18} \mathrm{~N}_{2} \mathrm{O}_{4} \mathrm{~S} \cdot \mathrm{C}_{13} \mathrm{H}_{20} \mathrm{~N}_{2} \mathrm{O}_{2} \cdot \mathrm{H}_{2} \mathrm{O}\right)$, benzathine penicillin $\left[\mathrm{C}_{16} \mathrm{H}_{20} \mathrm{~N}_{2} \cdot\left(\mathrm{C}_{16} \mathrm{H}_{18} \mathrm{~N}_{2} \mathrm{O}_{4} \mathrm{~S}\right)_{2}\right]$ benzyl penicillin sodium $\left(\mathrm{C}_{16} \mathrm{H}_{17} \mathrm{~N}_{2} \mathrm{NaO}_{4} \mathrm{~S}\right)$, cefalexin $\left(\mathrm{C}_{16} \mathrm{H}_{17} \mathrm{~N}_{3} \mathrm{O}_{4} \mathrm{~S} \cdot \mathrm{H}_{2} \mathrm{O}\right)$, cefotaxime sodium $\left[\mathrm{C}_{15} \mathrm{H}_{16} \mathrm{~N}_{5} \mathrm{NaO}_{2} \mathrm{~S} \cdot 3(1 / 2) \mathrm{H}_{2} \mathrm{O}\right]$, ceftriaxone sodium $\left[\mathrm{C}_{18} \mathrm{H}_{16} \mathrm{~N}_{8} \mathrm{Na}_{2} \mathrm{O}_{7} \mathrm{~S}_{3} \cdot 3(1 / 2) \mathrm{H}_{2} \mathrm{O}\right]$, doxycycline hydrochloride $\left(\mathrm{C}_{22} \mathrm{H}_{24} \mathrm{~N}_{2} \mathrm{O}_{8} \cdot \mathrm{HCl} \cdot 1 / 2 \mathrm{C}_{2} \mathrm{H}_{5} \mathrm{OH} \cdot 1 / 2 \mathrm{H}_{2} \mathrm{O}\right), \quad$ oxytetracycline dihydrate $\left(\mathrm{C}_{22} \mathrm{H}_{24} \mathrm{~N}_{2} \mathrm{O}_{9} \cdot 2 \mathrm{H}_{2} \mathrm{O}\right)$, tetracycline hydrochloride $\left(\mathrm{C}_{22} \mathrm{H}_{24} \mathrm{~N}_{2} \mathrm{O}_{8} \cdot \mathrm{HCl}\right)$, azithromycin $\left(\mathrm{C}_{38} \mathrm{H}_{72} \mathrm{~N}_{2} \mathrm{O}_{12}\right)$ and erythromycin estolate $\left(\mathrm{C}_{40} \mathrm{H}_{71} \mathrm{NO}_{14} \cdot \mathrm{C}_{12} \mathrm{H}_{26} \mathrm{O}_{4} \mathrm{~S}\right)$ antibiotics were 
Table 1 X-ray diffraction data in terms of lattice spacing and relative intensities for certain $\beta$-lactam antibiotic drugs

\begin{tabular}{|c|c|c|c|c|c|c|c|c|c|c|c|c|c|c|c|}
\hline \multicolumn{2}{|c|}{ Ampicillin $\mathrm{Na}$} & \multicolumn{2}{|c|}{$\begin{array}{c}\text { Ampicillin. } \\
\qquad 3 \mathrm{H}_{2} \mathrm{O}\end{array}$} & \multicolumn{2}{|c|}{$\begin{array}{c}\text { Penicillin } G \\
\text { procaine }\end{array}$} & \multicolumn{2}{|c|}{$\begin{array}{l}\text { Benzathine } \\
\text { penicillin }\end{array}$} & \multicolumn{2}{|c|}{$\begin{array}{c}\text { Benzyl } \\
\text { penicillin·Na }\end{array}$} & \multicolumn{2}{|c|}{ Cefalexin } & \multicolumn{2}{|c|}{ Ceftriaxone $\cdot \mathrm{Na}$} & \multicolumn{2}{|c|}{ Cefotaxime $\cdot \mathrm{Na}$} \\
\hline$\AA$ & $I / I_{\mathrm{I}}$ & $\AA$ & $I / I_{\mathrm{I}}$ & $\AA$ & $I / I_{\mathrm{I}}$ & $\AA$ & $I / I_{\mathrm{I}}$ & $\AA$ & $I / I_{\mathrm{I}}$ & $\AA$ & $I / I_{\mathrm{I}}$ & $\AA$ & $I / I_{\mathrm{I}}$ & $\AA$ & $I / I_{\mathrm{I}}$ \\
\hline 6.52 & 13.75 & 7.18 & 82.57 & 8.38 & 18.39 & 7.87 & 17.80 & 5.97 & 51.45 & 8.04 & 36.03 & 7.98 & 85.80 & 7.96 & 62.77 \\
\hline 5.87 & 15.55 & 5.83 & 100.00 & 7.46 & 13.24 & 7.23 & 100.00 & 5.83 & 17.90 & 7.01 & 12.72 & 7.11 & 25.18 & 7.10 & 26.80 \\
\hline 5.56 & 63.00 & 5.16 & 23.35 & 7.05 & 27.24 & 6.77 & 16.68 & 5.22 & 100.00 & 6.83 & 11.86 & 6.39 & 18.36 & 5.75 & 29.18 \\
\hline 5.12 & 100.00 & 4.89 & 95.60 & 6.60 & 21.02 & 6.33 & 78.75 & 5.06 & 22.61 & 6.59 & 12.09 & 5.76 & 30.81 & 5.30 & 20.62 \\
\hline 4.72 & 49.88 & 4.54 & 56.42 & 6.07 & 23.56 & 5.88 & 31.99 & 4.87 & 19.21 & 6.07 & 38.66 & 5.09 & 14.44 & 4.84 & 38.08 \\
\hline 4.46 & 60.37 & 4.08 & 22.86 & 5.80 & 13.34 & 5.54 & 35.30 & 4.77 & 8.23 & 5.59 & 29.18 & 4.84 & 43.09 & 4.72 & 24.26 \\
\hline 4.19 & 30.49 & 3.99 & 52.29 & 5.59 & 26.54 & 5.11 & 14.02 & 4.59 & 8.22 & 5.42 & 100.00 & 4.65 & 43.22 & 4.65 & 34.82 \\
\hline 3.80 & 49.21 & 3.81 & 56.27 & 5.23 & 21.59 & 4.85 & 20.92 & 4.34 & 80.98 & 5.29 & 15.64 & 4.45 & 100.00 & 4.45 & 100.00 \\
\hline 3.54 & 9.38 & 3.75 & 69.48 & 4.90 & 17.51 & 4.71 & 23.49 & 4.19 & 44.61 & 4.97 & 42.30 & 4.20 & 37.83 & 4.22 & 42.44 \\
\hline 3.42 & 22.08 & 3.59 & 5.77 & 4.77 & 29.19 & 4.65 & 35.04 & 4.06 & 10.77 & 4.89 & 13.70 & 3.95 & 44.10 & 4.20 & 37.41 \\
\hline 3.21 & 16.37 & 3.50 & 30.97 & 4.73 & 42.14 & 4.53 & 28.33 & 4.02 & 7.15 & 4.75 & 15.80 & 3.92 & 59.03 & 3.92 & 53.19 \\
\hline 2.98 & 18.68 & 3.47 & 64.15 & 4.64 & 100.00 & 4.48 & 36.51 & 3.91 & 2.24 & 4.56 & 36.88 & 3.74 & 45.86 & 3.75 & 41.18 \\
\hline 2.73 & 14.32 & 3.31 & 65.17 & 4.59 & 21.06 & 4.34 & 11.37 & 3.65 & 33.61 & 4.37 & 25.36 & 3.71 & 26.35 & 3.71 & 43.09 \\
\hline 2.53 & 12.20 & 3.22 & 21.37 & 4.49 & 15.07 & 4.18 & 51.95 & 3.57 & 4.97 & 4.22 & 31.47 & 3.60 & 48.94 & 3.60 & 77.94 \\
\hline 2.35 & 5.14 & 3.07 & 59.79 & 4.29 & 13.85 & 3.94 & 27.55 & 3.48 & 12.92 & 4.02 & 53.78 & 3.55 & 20.10 & 3.55 & 17.82 \\
\hline 2.19 & 4.63 & 2.96 & 19.11 & 4.13 & 40.00 & 3.88 & 31.49 & 3.42 & 13.29 & 3.86 & 39.74 & 3.33 & 46.16 & 3.42 & 9.43 \\
\hline 2.05 & 5.61 & 2.80 & 36.10 & 4.00 & 5.01 & 3.74 & 16.64 & 3.33 & 29.58 & 3.77 & 6.54 & 3.23 & 13.49 & 3.32 & 39.83 \\
\hline 1.94 & 8.37 & 2.74 & 17.50 & 3.89 & 5.80 & 3.65 & 40.17 & 3.16 & 25.77 & 3.69 & 14.43 & 3.17 & 32.56 & 3.22 & 18.64 \\
\hline 1.85 & 7.43 & 2.68 & 17.08 & 3.73 & 17.78 & 3.62 & 57.76 & 3.00 & 90.46 & 3.63 & 20.60 & 3.09 & 9.93 & 3.17 & 26.55 \\
\hline \multirow[t]{36}{*}{1.62} & 1.48 & 2.57 & 20.04 & 3.69 & 26.52 & 3.49 & 16.04 & 2.91 & 5.55 & 3.57 & 17.82 & 3.03 & 30.98 & 3.09 & 17.65 \\
\hline & & 2.51 & 19.73 & 3.64 & 7.50 & 3.43 & 16.11 & 2.81 & 26.22 & 3.44 & 9.01 & 2.93 & 8.98 & 3.03 & 33.40 \\
\hline & & 2.39 & 5.30 & 3.57 & 14.14 & 3.27 & 28.19 & 2.80 & 20.94 & 3.41 & 15.69 & 2.65 & 22.86 & 2.94 & 10.53 \\
\hline & & 2.26 & 27.98 & 3.53 & 26.93 & 3.19 & 24.46 & 2.71 & 6.31 & 3.35 & 7.48 & 2.59 & 5.59 & 2.84 & 6.56 \\
\hline & & 2.21 & 11.53 & 3.38 & 14.16 & 3.16 & 22.50 & 2.61 & 30.72 & 3.28 & 10.94 & 2.52 & 11.93 & 2.65 & 26.75 \\
\hline & & 2.15 & 27.82 & 3.31 & 20.27 & 3.10 & 30.44 & 2.54 & 50.09 & 3.24 & 16.37 & 2.37 & 9.95 & 2.61 & 11.66 \\
\hline & & 2.10 & 17.66 & 3.27 & 23.94 & 2.99 & 11.55 & 2.41 & 8.71 & 3.18 & 10.27 & 2.34 & 9.01 & 2.51 & 15.24 \\
\hline & & 2.03 & 54.12 & 3.16 & 7.36 & 2.88 & 39.91 & 2.29 & 5.42 & 3.11 & 32.04 & 2.31 & 12.13 & 2.35 & 11.60 \\
\hline & & 1.95 & 16.40 & 3.12 & 9.50 & 2.82 & 14.57 & 2.24 & 10.23 & 2.99 & 10.22 & 2.23 & 6.44 & 2.31 & 14.54 \\
\hline & & 1.84 & 11.05 & 3.03 & 10.26 & 2.75 & 9.81 & 2.20 & 4.82 & 2.91 & 11.98 & 2.18 & 11.65 & 2.26 & 7.29 \\
\hline & & 1.77 & 2.90 & 2.97 & 12.94 & 2.71 & 6.73 & 2.11 & 15.04 & 2.88 & 7.79 & 2.10 & 5.19 & 2.17 & 8.77 \\
\hline & & 1.73 & 7.17 & 2.88 & 4.56 & 2.67 & 13.27 & 2.04 & 11.89 & 2.81 & 5.16 & 2.05 & 14.35 & 2.10 & 7.12 \\
\hline & & 1.67 & 7.45 & 2.82 & 5.07 & 2.57 & 17.08 & 1.99 & 3.47 & 2.73 & 12.48 & 1.99 & 5.33 & 2.05 & 14.08 \\
\hline & & 1.61 & 6.12 & 2.71 & 4.79 & 2.51 & 7.26 & 1.96 & 5.29 & 2.70 & 12.48 & 1.89 & 4.83 & 1.99 & 4.35 \\
\hline & & 1.43 & 5.45 & 2.63 & 4.35 & 2.47 & 7.72 & 1.94 & 5.51 & 2.66 & 5.88 & 1.86 & 4.80 & 1.89 & 5.30 \\
\hline & & 1.40 & 3.60 & 2.60 & 4.55 & 2.41 & 11.20 & 1.88 & 8.29 & 2.62 & 4.88 & 1.84 & 5.48 & 1.86 & 4.89 \\
\hline & & & & 2.46 & 17.35 & 2.25 & 7.66 & 1.85 & 8.93 & 2.59 & 3.79 & 1.80 & 1.55 & 1.84 & 5.30 \\
\hline & & & & 2.37 & 3.40 & 2.19 & 10.52 & 1.83 & 3.51 & 2.54 & 2.54 & 1.67 & 1.20 & 1.66 & 1.34 \\
\hline & & & & 2.34 & 6.46 & 2.17 & 4.92 & 1.81 & 2.29 & 2.46 & 4.84 & 1.63 & 0.56 & 1.42 & 1.30 \\
\hline & & & & 2.29 & 6.02 & 2.11 & 6.46 & 1.74 & 1.40 & 2.43 & 5.53 & 1.61 & 0.66 & & \\
\hline & & & & 2.26 & 3.17 & 2.06 & 15.98 & 1.69 & 8.32 & 2.38 & 4.55 & 1.54 & 2.18 & & \\
\hline & & & & 2.22 & 4.55 & 1.96 & 3.58 & 1.66 & 2.19 & 2.29 & 2.04 & 1.51 & 1.14 & & \\
\hline & & & & 2.20 & 5.08 & 1.91 & 5.61 & 1.65 & 1.85 & 2.24 & 5.36 & 1.49 & 1.17 & & \\
\hline & & & & 2.15 & 3.59 & 1.83 & 4.78 & 1.59 & 0.71 & 2.13 & 3.79 & 1.40 & 1.47 & & \\
\hline & & & & 2.05 & 4.90 & 1.78 & 3.70 & 1.52 & 3.43 & 2.07 & 3.95 & & & & \\
\hline & & & & 2.02 & 5.85 & 1.75 & 2.46 & 1.48 & 1.11 & 1.89 & 1.35 & & & & \\
\hline & & & & 1.94 & 2.16 & 1.68 & 1.87 & 1.42 & 1.70 & 1.86 & 1.59 & & & & \\
\hline & & & & 1.92 & 2.43 & 1.64 & 2.01 & 1.36 & 0.70 & 1.82 & 0.90 & & & & \\
\hline & & & & 1.88 & 1.82 & 1.58 & 1.63 & & & 1.79 & 1.17 & & & & \\
\hline & & & & 1.82 & 1.94 & 1.52 & 1.02 & & & 1.75 & 0.51 & & & & \\
\hline & & & & 1.79 & 1.68 & 1.45 & 1.18 & & & 1.71 & 0.87 & & & & \\
\hline & & & & 1.75 & 1.41 & 1.44 & 0.92 & & & 1.68 & 1.14 & & & & \\
\hline & & & & 1.70 & 1.75 & 1.41 & 0.63 & & & 1.65 & 0.52 & & & & \\
\hline & & & & 1.64 & 0.96 & & & & & & & & & & \\
\hline & & & & 1.58 & 0.93 & & & & & & & & & & \\
\hline & & & & 1.44 & 0.30 & & & & & & & & & & \\
\hline
\end{tabular}

provided as gift samples by Central Drugs Laboratory, Calcutta and Karnataka Antibiotics \& Pharmaceuticals Ltd., Bangalore.

\section{Apparatus}

XRD measurements were obtained using the Philips X'pert on a powder diffraction system (Philips Analytical, The Netherlands) equipped with a vertical goniometer in the BraggBrentano focusing geometry. The $\mathrm{X}$-ray generator was operated at $40 \mathrm{kV}$ and $50 \mathrm{~mA}$, using the $\mathrm{Cu} K_{\alpha}$ line at $1.54056 \AA$ as the radiation source. Each sample was scanned from $10^{\circ}$ to $70^{\circ}(2 \theta)$ and in step sizes of 0.020 , count time of $2.00 \mathrm{~s}$, using an automatic divergence slit assembly and a proportional detector. The samples were scanned at $25^{\circ} \mathrm{C}$. Relative intensities were read from the strip charts and corrected to fixed the slit values.

\section{Operation procedure}

A powdered specimen is usually packed and prepared in a specimen holder made of aluminum or glass. In setting up the specimen and apparatus, the coplanarity of the specimen surface with the specimen holder surface and the setting of the specimen holder at the position of symmetric reflection geometry have to be assured. The powders were passed through a 100 mesh sieve and placed into the sample holder by the side drift technique. ${ }^{22}$ The holder consisted of a central cavity. In order to prepare a sample for analysis, a glass slide was clipped up to the top face of the sample holder so as to form a wall. Each powder was filled into the holder, gently tapped and used for the XRD analysis.

\section{Results and Discussion}

Table 1 gives the data obtained for the eight $\beta$-lactam and Table 2 for the three tetracycline and two macrolide antibiotic drugs in terms of the lattice spacing and the relative line intensities. Because most of the characteristic lines in the diffraction patterns were generally prominent and sharp, the measurements of the angles, and hence of the $d$-values were accurate. Proper sample preparation helped to attain accurate peak positions for qualitative analysis. If the sample surface was irregular, or if it 
Table 2 X-ray diffraction data in terms of lattice spacing and relative intensities for certain tetracycline and macrolide antibiotic drugs

\begin{tabular}{|c|c|c|c|c|c|c|c|c|c|}
\hline \multicolumn{2}{|c|}{$\begin{array}{c}\text { Tetracycline. } \\
\mathrm{HCl}\end{array}$} & \multicolumn{2}{|c|}{$\begin{array}{c}\text { Doxycycline. } \\
\mathrm{HCl}\end{array}$} & \multicolumn{2}{|c|}{$\begin{array}{c}\text { Oxytetracycline } \\
.2 \mathrm{H}_{2} \mathrm{O}\end{array}$} & \multicolumn{2}{|c|}{ Azithromycin } & \multicolumn{2}{|c|}{$\begin{array}{c}\text { Erythromycin } \\
\text { estolate }\end{array}$} \\
\hline$\AA$ & $I / I_{\mathrm{I}}$ & $\AA$ & $I / I_{\mathrm{I}}$ & $\AA$ & $I / I_{\mathrm{I}}$ & $\AA$ & $I / I_{\mathrm{I}}$ & $\AA$ & $I / I_{\mathrm{I}}$ \\
\hline 8.38 & 39.47 & 8.08 & 100.00 & 8.26 & 79.90 & 7.53 & 22.94 & 8.27 & 34.51 \\
\hline 7.91 & 74.11 & 7.95 & 62.31 & 8.01 & 100.00 & 7.05 & 28.57 & 7.49 & 29.50 \\
\hline 7.40 & 43.41 & 7.41 & 28.48 & 7.48 & 80.09 & 6.90 & 85.71 & 6.95 & 38.64 \\
\hline 6.76 & 36.82 & 7.21 & 30.07 & 6.35 & 59.01 & 5.73 & 100.00 & 6.64 & 43.18 \\
\hline 6.43 & 59.28 & 6.90 & 20.63 & 6.09 & 58.35 & 5.64 & 28.71 & 6.46 & 33.15 \\
\hline 5.96 & 25.16 & 6.58 & 15.66 & 5.80 & 71.48 & 5.48 & 37.71 & 6.18 & 26.89 \\
\hline 5.74 & 57.16 & 6.30 & 16.67 & 5.38 & 65.98 & 5.40 & 27.47 & 5.44 & 29.07 \\
\hline 5.56 & 100.00 & 6.09 & 56.29 & 4.97 & 61.74 & 5.11 & 11.75 & 5.25 & 58.53 \\
\hline 5.24 & 45.46 & 5.86 & 49.20 & 4.49 & 74.99 & 4.83 & 88.65 & 5.15 & 100.00 \\
\hline 4.82 & 49.10 & 5.70 & 27.95 & 4.45 & 82.78 & 4.79 & 71.81 & 4.80 & 30.26 \\
\hline 4.54 & 43.67 & 5.48 & 27.22 & 4.39 & 61.12 & 4.67 & 10.91 & 4.69 & 44.64 \\
\hline 4.47 & 56.47 & 5.38 & 16.39 & 4.07 & 72.71 & 4.52 & 33.48 & 4.48 & 36.36 \\
\hline 4.45 & 60.36 & 5.17 & 15.69 & 4.05 & 73.65 & 4.42 & 21.85 & 4.27 & 30.40 \\
\hline 4.26 & 57.94 & 5.05 & 26.43 & 3.99 & 55.39 & 4.37 & 19.78 & 4.14 & 20.65 \\
\hline 4.13 & 39.17 & 4.96 & 30.87 & 3.78 & 86.51 & 4.28 & 22.60 & 4.05 & 18.90 \\
\hline 4.06 & 43.86 & 4.83 & 13.36 & 3.72 & 57.40 & 4.19 & 52.03 & 3.93 & 12.11 \\
\hline 4.04 & 48.94 & 4.69 & 29.67 & 3.62 & 40.04 & 4.14 & 47.38 & 3.81 & 14.51 \\
\hline 3.95 & 19.33 & 4.51 & 29.15 & 3.44 & 45.08 & 4.10 & 27.56 & 3.64 & 14.75 \\
\hline 3.82 & 40.77 & 4.39 & 27.42 & 3.36 & 47.39 & 3.72 & 28.17 & 3.44 & 14.98 \\
\hline 3.69 & 40.07 & 4.31 & 24.56 & 3.29 & 51.01 & 3.63 & 17.31 & 3.30 & 6.89 \\
\hline 3.64 & 45.38 & 4.11 & 22.85 & 3.22 & 50.91 & 3.43 & 36.28 & 3.18 & 6.85 \\
\hline 3.57 & 31.28 & 3.98 & 44.01 & 3.17 & 48.72 & 3.34 & 23.65 & 2.70 & 3.06 \\
\hline 3.33 & 23.26 & 3.92 & 47.71 & 3.11 & 53.28 & 3.18 & 19.12 & 2.61 & 1.70 \\
\hline 3.28 & 43.56 & 3.88 & 36.21 & 3.08 & 53.36 & 2.96 & 53.97 & 2.33 & 1.68 \\
\hline 3.22 & 41.92 & 3.74 & 39.53 & 2.89 & 47.91 & 2.93 & 22.24 & 2.28 & 2.44 \\
\hline 3.12 & 63.44 & 3.70 & 26.07 & 2.84 & 32.93 & 2.81 & 12.72 & 2.15 & 1.57 \\
\hline 3.03 & 8.58 & 3.61 & 97.26 & 2.79 & 33.86 & 2.74 & 5.21 & 2.03 & 11.49 \\
\hline 2.96 & 28.16 & 3.48 & 33.77 & 2.74 & 32.06 & 2.67 & 10.26 & 1.90 & 1.51 \\
\hline 2.87 & 16.09 & 3.44 & 31.33 & 2.66 & 29.38 & 2.65 & 44.46 & 1.65 & 2.79 \\
\hline 2.85 & 15.49 & 3.36 & 12.20 & 2.56 & 37.21 & 2.53 & 11.87 & & \\
\hline 2.81 & 14.52 & 3.33 & 18.36 & 2.54 & 61.67 & 2.45 & 44.49 & & \\
\hline 2.74 & 38.06 & 3.23 & 28.76 & 2.48 & 23.30 & 2.44 & 46.30 & & \\
\hline 2.68 & 18.66 & 3.17 & 14.85 & 2.39 & 22.97 & 2.43 & 28.78 & & \\
\hline 2.63 & 15.57 & 3.09 & 10.79 & 2.30 & 28.09 & 2.36 & 14.11 & & \\
\hline 2.57 & 16.41 & 3.02 & 12.53 & 2.28 & 27.65 & 2.31 & 14.51 & & \\
\hline 2.54 & 20.43 & 2.97 & 13.91 & 2.19 & 19.69 & 2.21 & 19.62 & & \\
\hline 2.48 & 16.59 & 2.93 & 14.66 & 2.14 & 14.92 & 2.20 & 35.88 & & \\
\hline 2.43 & 15.72 & 2.84 & 11.04 & 2.09 & 19.91 & 2.15 & 23.95 & & \\
\hline 2.40 & 12.20 & 2.77 & 16.96 & 2.03 & 30.22 & 2.10 & 8.11 & & \\
\hline 2.31 & 7.51 & 2.65 & 21.04 & 2.02 & 22.00 & 2.03 & 35.33 & & \\
\hline 2.24 & 12.31 & 2.60 & 10.24 & 1.96 & 11.03 & 1.94 & 14.51 & & \\
\hline 2.22 & 11.92 & 2.55 & 17.19 & 1.89 & 9.50 & 1.78 & 3.27 & & \\
\hline 2.20 & 16.68 & 2.53 & 16.19 & 1.86 & 6.30 & 1.72 & 5.29 & & \\
\hline 2.17 & 10.37 & 2.46 & 9.54 & 1.80 & 6.68 & 1.69 & 2.67 & & \\
\hline 2.13 & 12.01 & 2.41 & 10.12 & 1.76 & 6.65 & 1.63 & 2.45 & & \\
\hline 2.09 & 11.86 & 2.37 & 9.40 & 1.71 & 2.89 & 1.59 & 2.53 & & \\
\hline 2.07 & 11.31 & 2.29 & 11.20 & 1.65 & 5.95 & 1.57 & 1.96 & & \\
\hline 2.00 & 9.11 & 2.25 & 9.70 & 1.58 & 4.81 & 1.56 & 2.85 & & \\
\hline 1.94 & 7.41 & 2.21 & 9.00 & & & 1.54 & 2.87 & & \\
\hline 1.88 & 8.82 & 2.16 & 13.46 & & & & & & \\
\hline 1.84 & 5.19 & 2.07 & 9.30 & & & & & & \\
\hline 1.80 & 6.25 & 2.03 & 5.50 & & & & & & \\
\hline 1.77 & 4.05 & 1.98 & 10.34 & & & & & & \\
\hline 1.74 & 8.10 & 1.93 & 5.42 & & & & & & \\
\hline 1.68 & 5.38 & 1.90 & 5.25 & & & & & & \\
\hline 1.60 & 2.39 & 1.88 & 6.02 & & & & & & \\
\hline 1.56 & 0.57 & 1.86 & 4.68 & & & & & & \\
\hline 1.50 & 0.88 & 1.81 & 3.49 & & & & & & \\
\hline \multirow[t]{6}{*}{1.42} & 1.18 & 1.79 & 3.10 & & & & & & \\
\hline & & 1.76 & 1.78 & & & & & & \\
\hline & & 1.71 & 3.44 & & & & & & \\
\hline & & 1.70 & 2.81 & & & & & & \\
\hline & & 1.67 & 0.66 & & & & & & \\
\hline & & 1.50 & 0.76 & & & & & & \\
\hline
\end{tabular}

was displaced from the focusing circle, the peak locations and intensities would vary.

Powder X-ray diffraction patterns for these drugs are given Figs. 4-7. All of the high-intensity lines (relative intensity) observed in the powder pattern of these drugs were observed in the pure form (Tables 1 and 2). The diffraction patterns of lowabsorption drugs measured in para focusing Bragg-Brentano geometry exhibit severe line broadening. Identification of a structure from its powdered diffraction pattern is based upon the position of lines and their relative intensities. Each powder pattern is characterized by the interplanar $d$ spacing and the relative intensities $\left(I / I_{\mathrm{I}}\right)$ of the three strongest lines in the pattern under the Hanawalt system.

In this study, ampicillin was used as the model compound. The USP lists the ampicillin sodium and trihydrate forms of ampicillin. Marketed ampicillin capsules contain either their anhydrate or the trihydrate, ${ }^{23}$ and these two forms exhibit pronounced differences in their XRD patterns (Fig. 4). A comparison of the two XRD patterns reveals that, in the angular range where some peaks of ampicillin sodium occur, there are no peaks of ampicillin trihydrate, and vice versa. ${ }^{24,25}$

Every crystalline powder produces a characteristic diffraction pattern. This is the basis of qualitative analysis by powder diffraction. Identification is usually accomplished by a systematic comparison of an unknown pattern with a catalogue of standard data, such as the powder diffraction file published by the International Center for Diffraction Data (ICDD). The first effort was directed towards the identification of ampicillin sodium in a capsule formulation. Before analysis of the formulations, the XRD patterns of anhydrous ampicillin were experimentally obtained and compared with the ICDD card pattern. There was in general good agreement between the two. However, several lines were missing in the XRD pattern obtained in this work. The XRD pattern of ampicillin trihydrate was experimentally obtained and compared with the ICDD card pattern. ${ }^{25}$

Nevertheless, complete diffraction patterns are still characteristics can be seen from Tables 1 and 2 . An examination of the X-ray data shows that a compound can be easily distinguished from other members. Despite the fact that the eight $\beta$-lactams, three tetracyclines and two macrolide antibiotic drugs have almost the same molecular structure in their respective groups, their powder diffraction patterns are surprisingly different (Figs. 4-7). In general, there are only three or four of the most intense lines that are important for characterizing the compound.

A comparison of the relative intensities of the X-ray lines does not reveal good agreement. The intensity of the X-ray lines can be affected by several factors, including preferred orientation. Any sample-induced line broadening would make it difficult to distinguish between lines with close $d$-spacing values. There are also several instrumental factors that influence the peak resolution. ${ }^{26}$

However, the proposed method does not have any inherent difficulty originating from this fact. The number of emerging peaks is too much, and the overlap of the several peaks may influence the accuracy of the relative peak intensities. Since the goal of the project is phase identification, our predominant interest is the position of X-ray lines ( $d$ spacing).

A comparison of the XRD pattern of $\beta$-lactam reveals marked differences in the angular ranges and $d$ spacing: $7.98 \AA$ $\left(11.08^{\circ} 2 \theta\right)$ and $2.54 \AA\left(35.29^{\circ} 2 \theta\right)$. The XRD pattern of tetracyclines shows angular ranges and $d$ spacing of $8.38 \AA$ $\left(10.55^{\circ} 2 \theta\right)$ and $2.89 \AA\left(30.95^{\circ} 2 \theta\right)$. The XRD pattern of macrolides shows angular ranges and $d$ spacing of $8.27 \AA$ $\left(10.69^{\circ} 2 \theta\right)$ and $3.43 \AA\left(25.97^{\circ} 2 \theta\right)$. In the above-mentioned groups, each of the drugs in their respective groups has a distinct $d$ spacing and angular ranges. For example, in the macrolide group azithromycin has $7.53 \AA\left(11.75^{\circ} 2 \theta\right)$ and $3.43 \AA$ $\left(25.97^{\circ} 2 \theta\right)$, but erythromycin has $8.27 \AA\left(10.69^{\circ} 2 \theta\right)$ and $3.44 \AA$ $\left(25.91^{\circ} 2 \theta\right)$. Therefore, our discussion is restricted to the $d$ spacing of the X-ray line. However, the relative intensities of the lines are also probable.

XRD has the potential ability to identify not only the active ingredient, but also the crystalline excipients in a formulation. 

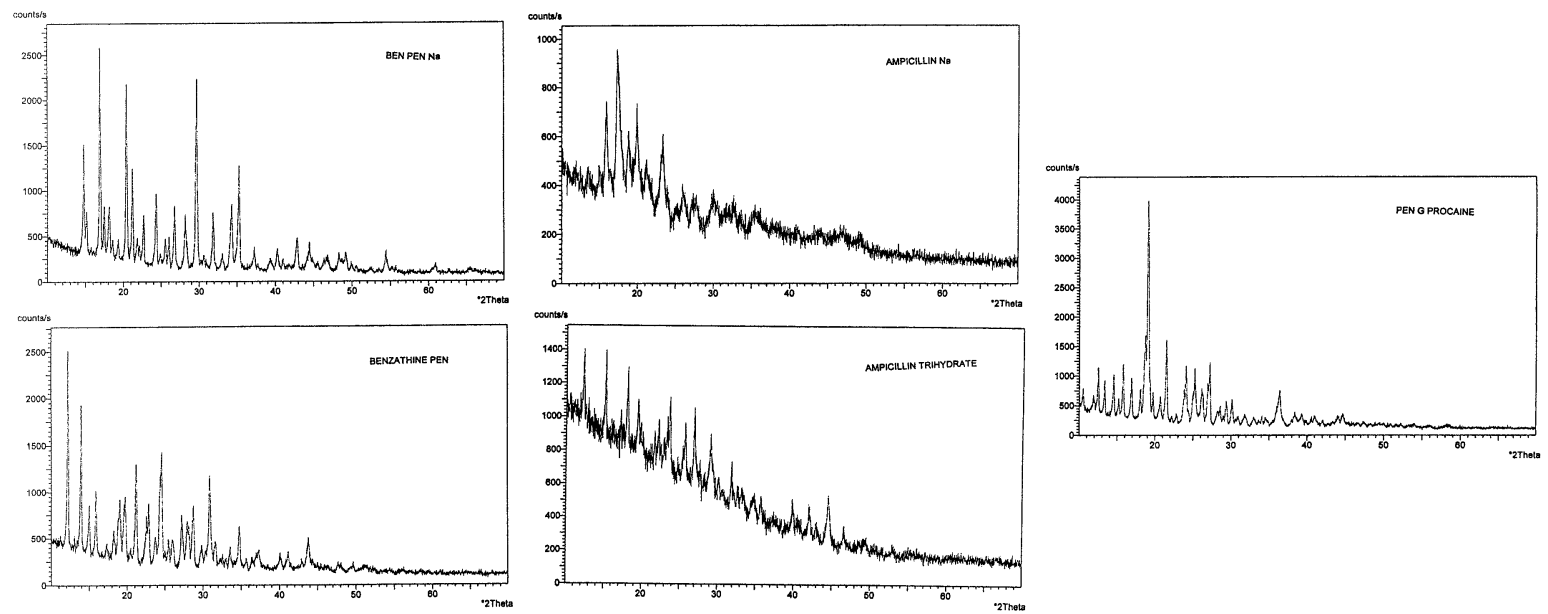

Fig. 4 XRD pattern of $\beta$-lactam antibiotics.
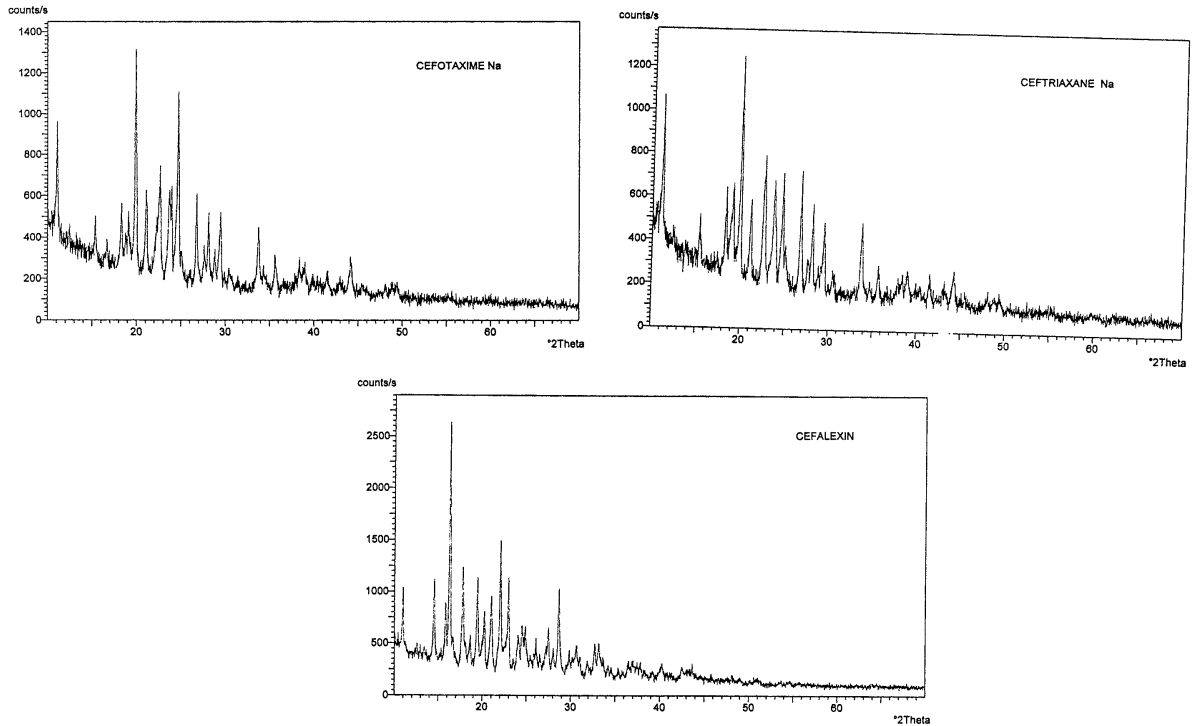

Fig. 5 XRD pattern of cephalosporin antibiotics.

The $\beta$-lactam, tetracycline and macrolide antibiotic drugs under investigation should not only be distinguished one from the other, but from most components of the pharmaceutical preparations. The aim of this paper is to produce a basis for powder XRD as a promising new official method for the identification of these antibiotic drugs from formulations and forensic samples. Our current findings provide preliminary data, which make the basis for validating the new method.

\section{Conclusion}

X-ray powder diffraction data have been developed or refined for the identification of drugs, excipients, and adulterants found in illicit preparations. The powder XRD patterns for the eight $\beta$-lactam, three tetracycline and two macrolide antibiotic drugs studied were sufficiently unique to make their identification possible.

The current preliminary studies make the basis for the validation of powder XRD as a new monitoring official method for the identification of $\beta$-lactam, tetracycline and macrolide antibiotic drugs.

\section{Acknowledgements}

The authors are very grateful to Dr. C. N. Bhattacharya, Director, Central Forensic Science Laboratory, Directorate of Forensic Science, Hyderabad-13 for providing the laboratory facilities. The authors are indebted to Karnataka Antibiotics \& Pharmaceuticals Ltd., Bangalore and Central Drugs Laboratory, Calcutta for providing gift samples of pure $\beta$-lactam, tetracycline and macrolide antibiotic drugs.

\section{References}

1. R. K. Blackwood, J. J. Beereboom, H. H. Rennhard, M. Schach von Wittenau, and C. R. Stephens, J. Am. Chem. Soc., 1963, 85, 3943.

2. A. J. Bryskier, J. P. Butzler, H. C. Neu, and P. M. Tulkens, "Macrolides: Chemistry, Pharmacology and Clinical 

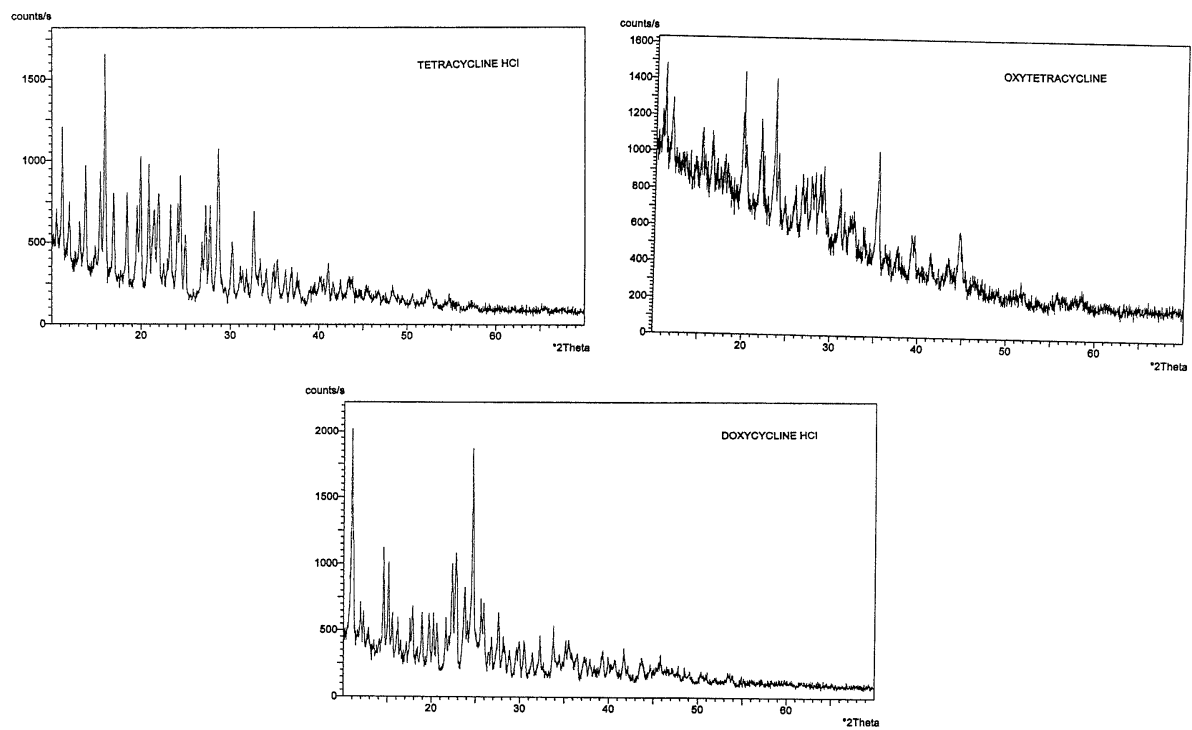

Fig. 6 XRD pattern of tetracycline antibiotics.
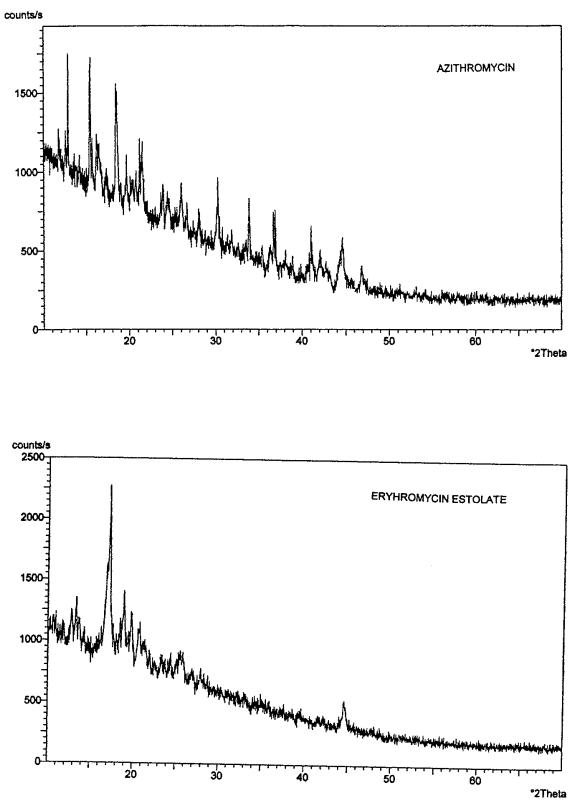

Fig. 7 XRD pattern of macrolide antibiotics.

Uses", 1993, Arnette Blackwell, Paris, France.

3. V. A. Folen, J. Forensic Sci., 1975, 20(2), 348.

4. J. D. Hanawalt, H. W. Rinn, and L. K. Frevel, Ind. Eng. Chem., Anal. Ed., 1938, 10, 457.

5. A. W. Hull, J. Am. Chem. Soc., 1919, 41, 1168.

6. J. W. Shell, J. Pharm. Sci., 1963, 52, 24.

7. A. P. Queredo, H. N. de Armas, and L. X. Marill, Powder Diffr., 1998, 13, 20.

8. Y. Che, J. Zheng, J. Hao, and L. Chu, Powder Diffr., 2001, $16,165$.

9. S. Hayashi and H. Toraya, Powder. Diffr., 2000, 15, 86.

10. J. E. Kountourellis, C. K. Markopoulou, F. A. Underwood, and B. Chapman, Talanta, 1991, 38, 233.

11. R. Caminiti, G. Ortaggi, R. A. Mazzei, P. Ballirano, and R.
Rizzi, Powder Diffr., 2000, 15, 108.

12. J. T. R. Owen, R. Sithiraks, and F. A. Underwood, J. Assoc. Off. Anal. Chem., 1972, 55(6), 1171.

13. R. Caminiti, G. Ortaggi, R. A. Mazzei, P. Ballirano, and R. Rizzi, Powder Diffr., 2000, 15, 112.

14. N. V. Phadnis, R. K. Cavatur, and R. Suryanarayanan, J. Pharm. Biomed. Anal., 1997, 15, 929.

15. H. N. de Arms, E. P. Fontdevila, and R. P. Hernandez, Powder Diffr., 1999, 14, 142.

16. S. Thangadurai, S. K. Shukla, A. K. Srivastava, and Y. Anjaneyulu, Acta Pharm., 2003, 53, 295.

17. P. J. Thatcher and G. P. Briner, Powder Diffr., 1986, 4, 320.

18. C. J. Curry, D. F. Rendle, and A. Rogers, J. Forensic Sci. Soc., 1982, 22, 173.

19. "The United States Pharmacopeia", XXIV, NF XXI, 2001, United States Pharmacopeial Convenion, Rockville, 107 204.

20. "British Pharmacopoeia", 1999, Her Majesty's Stationary Office, London, 368.

21. "Indian Pharmacopoeia", Addendum 2000, 1996, The Controller of Publication, Ministry of Health and Family Welfare, Govt. of India, New Delhi 840 - 868.

22. M. C. Morris, H. F. McMurdie, and E. H. Evans, "Standard X-Ray Diffraction Powder Patterns", Sect. 16, Data for 86 substances (Monographs/US National Bureau of Standards, 25), 1979, National Technical Information Service, Springfield, $1-5$.

23. "Physicians Desk Reference", 50th ed., 1966, Medical Economics, Oradell, NJ, 733, 1558, 2084 - 2086, 2764.

24. "Powder Diffraction File: Set 33", in "Organic Databook", ed. W. F. McClune, 1989, International Centre for Diffraction Data, Swarthmore, PA, 10.

25. "Powder Diffraction File: Set 29", in "Organic Databook", ed. W. F. McClune, 1987, International Centre for Diffraction Data, Swarthmore, PA, 15.

26. H. P. Klug and L. E. Alexander, "X-ray Diffraction Proc. for Polycrystalline and Amorphous Materials", 2nd ed., 1974, Wiley, NY, 135 - 167, 202 - 206, 539 - 543. 